Publ. Math. Debrecen

Manuscript (September 23, 2010 [vm])

\title{
Automorphisms of partial endomorphism semigroups
}

\author{
By J. Araújo, V. H. Fernandes, M. M. Jesus, V. Maltcev and J. D. Mitchell
}

\begin{abstract}
In this paper we propose a general recipe for calculating the automorphism groups of semigroups consisting of partial endomorphisms of relational structures over a finite set with a single $m$-ary relation for any $m \in \mathbb{N}$.

We use this recipe to determine the automorphism groups of the following semigroups: the full transformation semigroup, the partial transformation semigroup, and the symmetric inverse semigroup, the wreath product of two full transformation semigroups, the partial endomorphisms of any partially ordered set, the full spectrum of semigroups of partial mappings preserving or reversing a linear or circular order.
\end{abstract}

\section{Introduction and the Main Result}

A number of works in the literature are dedicated to calculating the automorphism groups of certain transformation semigroups. In an earlier paper [3], a method for calculating automorphism groups of some such objects is given. In this paper we prove a more general result and use it to find the automorphism group of several well-known transformation semigroups. In order to state our main result we must recall some definitions and introduce some notation.

We assume throughout the paper that $\Omega$ is a finite set. We denote the semigroup of all partial mappings on $\Omega$ under composition of functions by $P_{\Omega}$, the semigroup of total mappings on $\Omega$ by $T_{\Omega}$, the inverse semigroup of partial injective mappings by $I_{\Omega}$, and the group of permutations on $\Omega$ by $S_{\Omega}$. If $\Omega=$ $\{1,2, \ldots, m\}$, then we abbreviate $P_{\Omega}, T_{\Omega}, I_{\Omega}$, and $S_{\Omega}$ to $P_{m}, T_{m}, I_{m}$, and $S_{m}$, respectively. If $U$ is a subsemigroup of $P_{\Omega}$, then we let $\operatorname{Aut}(U)$ denote the group

Mathematics Subject Classification: 20M10, 20M20.

Key words and phrases: automorphisms, endomorphism semigroups, relations. 
of automorphisms of $U$. For any $a \in S_{\Omega}$ let $\phi_{a}: P_{\Omega} \longrightarrow P_{\Omega}$ be the inner automorphism of $P_{\Omega}$ associated to $a$, that is, $\phi_{a}$ is the function defined by $(f) \phi_{a}=a^{-1} f a$ $\left(f \in P_{\Omega}\right)$. Now, for any subgroup $H$ of $S_{\Omega}$ denote

$$
\operatorname{Inn}_{H}(U)=\left\{\phi_{a} \mid a \in H \text { and }(U) \phi_{a}=U\right\} .
$$

In particular, denote by $\operatorname{Inn}(U)$ the group of inner automorphisms $\operatorname{Inn}_{S_{\Omega}}(U)$ of $U$.

The image of $f \in P_{\Omega}$ is denoted by $\operatorname{im}(f)$ and the domain of $f$ by $\operatorname{dom}(f)$. A mapping $f \in P_{\Omega}$ is called a constant with value $\alpha$ if $\beta f=\alpha$ for all $\beta \in \operatorname{dom}(f)$. For the sake of convenience, we will assume that the empty mapping $\emptyset$ is also a constant.

Let $m \in \mathbb{N}$. Then an $m$-ary relation $\rho$ on $\Omega$ is just a subset of

$$
\Omega^{m}=\left\{\left(\alpha_{1}, \alpha_{2}, \ldots, \alpha_{m}\right) \mid \alpha_{1}, \alpha_{2}, \ldots, \alpha_{m} \in \Omega\right\}
$$

If $\rho$ is an $m$-ary relation, then define

$$
\rho^{\prime}=\left\{\left(\alpha_{1}, \alpha_{2}, \ldots, \alpha_{m}\right) \in \rho \mid \alpha_{i} \neq \alpha_{j} \text { if } i \neq j\right\} .
$$

Let $\rho$ and $\sigma$ be $m$-ary relations on $\Omega$. We say that a subsemigroup $U$ of $P_{\Omega}$ acts transitively from $\rho^{\prime}$ to $\sigma^{\prime}$ if for all $\left(\alpha_{1}, \ldots, \alpha_{m}\right) \in \rho^{\prime}$ and $\left(\beta_{1}, \ldots, \beta_{m}\right) \in \sigma^{\prime}$ there exists $f \in U$ with

$$
\left(\alpha_{1} f, \ldots, \alpha_{m} f\right)=\left(\beta_{1}, \ldots, \beta_{m}\right) .
$$

If $U$ is a group of permutations and $\rho^{\prime}=\sigma^{\prime}=\left(\Omega^{m}\right)^{\prime}$, then our definition of transitivity is just the usual definition of $m$-transitivity for permutation groups. If $\left(\alpha_{1}, \ldots, \alpha_{m}\right) \in \Omega^{m}$ and $f \in U$, then we denote $\left(\alpha_{1} f, \ldots, \alpha_{m} f\right)$ by $\left(\alpha_{1}, \ldots, \alpha_{m}\right)^{f}$. The monoid of partial endomorphisms of $\rho$ is

$$
\operatorname{PEnd}(\rho)=\left\{f \in P_{\Omega} \mid\left(\alpha_{1}, \ldots, \alpha_{m}\right) \in \rho \cap \operatorname{dom}(f)^{m} \text { implies }\left(\alpha_{1}, \ldots, \alpha_{m}\right)^{f} \in \rho\right\} .
$$

We consider also the following submonoids of $\operatorname{PEnd}(\rho)$

$$
\operatorname{End}(\rho)=\operatorname{PEnd}(\rho) \cap T_{\Omega} \text { and } \operatorname{IEnd}(\rho)=\operatorname{PEnd}(\rho) \cap I_{\Omega}
$$

consisting of the (total) endomorphisms of $\rho$ and the partial injective endomorphisms of $\rho$, respectively.

If $\rho$ is a binary relation on $\Omega$, then an anti-automorphism of $\rho$ is an element $f \in S_{\Omega}$ such that $(\alpha, \beta) \in \rho$ implies $(\beta, \alpha)^{f} \in \rho$. Since $\Omega$ is finite, the set of 
automorphisms and anti-automorphisms of $\rho$ forms a group. The following definition can be thought of as a generalization of this notion to relations of higher arity. Let $H$ be a subgroup of $S_{m}$. Then we define

$$
\operatorname{Aut}_{H}(\rho)=\left\{f \in S_{\Omega} \mid(\exists t \in H)\left(\forall\left(\alpha_{1}, \ldots, \alpha_{m}\right) \in \rho\right)\left(\alpha_{(1) t}, \ldots, \alpha_{(m) t}\right)^{f} \in \rho\right\} .
$$

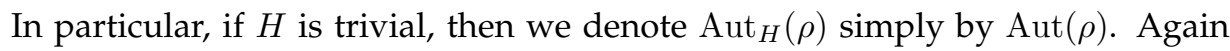
since $\Omega$ is finite, $\operatorname{Aut}_{H}(\rho)$ is a group. The group of automorphisms and antiautomorphisms of a binary relation $\rho$ is denoted by $\operatorname{Aut}_{S_{2}}(\rho)$ using this notation.

We also require the following definition:

$$
N(\rho, H)=\left\{\left(\alpha_{1}, \ldots, \alpha_{m}\right) \in\left(\Omega^{m}\right)^{\prime} \mid\left(\alpha_{(1) t}, \ldots, \alpha_{(m) t}\right) \notin \rho \text { for all } t \in H\right\} .
$$

We are now ready to state the main result of this paper, which is a generalization of [3, Theorem 2.1].

Theorem 1.1. Let $\rho$ be an $m$-ary relation on a finite set $\Omega$ for some $m \in \mathbb{N}$, let $H$ be a subgroup of $S_{m}$, and let $U$ be a subsemigroup of $\operatorname{PEnd}(\rho)$ such that:

(1) $U$ contains a constant idempotent with value $\alpha$ for all $\alpha \in \Omega$;

(2) $U$ acts transitively from $N(\rho, H) \cup \rho^{\prime}$ to $\rho^{\prime}$.

Then $\operatorname{Aut}(U)=\operatorname{Inn}_{\mathrm{Aut}_{H}\left(\rho^{\prime}\right)}(U)$. Moreover, if $a, b \in \operatorname{Aut}_{H}\left(\rho^{\prime}\right)$ are such that $a \neq b$ and $\phi_{a}, \phi_{b} \in \operatorname{Aut}(U)$, then $\phi_{a} \neq \phi_{b}$.

We prove Theorem 1.1 in Section 2. In Section 3, we derive several corollaries of Theorem 1.1, and discuss whether it is possible to weaken its hypothesis and still obtain its conclusion. In Sections 4 and 5, we use the main theorem and its corollaries to determine the automorphism groups of the following semigroups: the full transformation semigroup, the partial transformation semigroup, the symmetric inverse semigroup, the wreath product of two full transformation semigroups, the partial endomorphisms of any finite partially ordered set, the semigroups of partial mappings preserving or reversing a linear order, the semigroups of orientation-preserving or reversing partial mappings on a linear order, and related semigroups.

\section{Proof of Theorem 1.1}

We prove Theorem 1.1 in the following sequence of lemmas, some of them belonging to the folklore of this topic and included here for the sake of completeness. 
Lemma 2.1. Let $V$ be a subsemigroup of constants in $P_{\Omega}$ such that for all $\alpha \in$ $\Omega$ there exists a constant idempotent in $V$ with value $\alpha$. Then $\operatorname{Aut}(V)=\operatorname{Inn}(V)$.

Proof. The inclusion $\operatorname{Inn}(V) \leq \operatorname{Aut}(V)$ is obvious.

Let $\phi \in \operatorname{Aut}(V)$. If $\emptyset \in V$, then clearly $\emptyset \phi=\emptyset$. If $f \in V \backslash\{\emptyset\}$, then denote by $\alpha_{f}$ the unique element of $\operatorname{im}(f)$. Let $f, g \in V \backslash\{\emptyset\}$. It is obvious that if $f g \neq \emptyset$ then $\alpha_{(f g)}=\alpha_{g}$, and $f g \neq \emptyset$ if and only if $\alpha_{f} \in \operatorname{dom}(g)$. In particular, either $f^{2}=\emptyset$ or $f^{2}=f$, and if $h=h^{2} \in V \backslash\{\emptyset\}$ with $\alpha_{h}=\alpha_{f}$, then $f h=f$. Since $\phi \in \operatorname{Aut}(V), f g \neq \emptyset$ if and only if $(f \phi)(g \phi) \neq \emptyset$, so that $f g \neq \emptyset$ if and only if $\alpha_{f \phi} \in \operatorname{dom}(g \phi)$, and if $g=g^{2}$ and $f g \neq \emptyset$, then $(f \phi)(g \phi)=f \phi$.

By assumption, for all $\alpha \in \Omega$ there exists $h=h^{2} \in V$ such that $\alpha=\alpha_{h}$. We will show that $b: \Omega \longrightarrow \Omega$ defined by $(\alpha) b=\alpha_{h \phi}$ for all $\alpha \in \Omega$ is a well-defined bijection. If $f \in V \backslash\{\emptyset\}$ with $\alpha_{f}=\alpha$, then $f h=f \neq \emptyset$ and so $\alpha_{f \phi}=\alpha_{h \phi}$, so we have a well-defined mapping $b: \Omega \longrightarrow \Omega$. Suppose $\alpha b=\alpha^{\prime} b$ for some $\alpha, \alpha^{\prime} \in \Omega$. Then $\alpha_{h \phi}=\alpha_{h^{\prime} \phi}$ where $h, h^{\prime} \in V \backslash\{\emptyset\}$ are idempotents with $\alpha_{h}=\alpha$ and $\alpha_{h^{\prime}}=\alpha^{\prime}$. Since $\left(h h^{\prime}\right) \phi=(h \phi)\left(h^{\prime} \phi\right)=h \phi$, we have $h h^{\prime}=h$ and so $\alpha=\alpha_{h}=\alpha_{h^{\prime}}=\alpha^{\prime}$. Therefore $b$ is an injection and, since $\Omega$ is finite, we have $b \in S_{\Omega}$.

Let $f \in V \backslash\{\emptyset\}$. Take any $\alpha \in \Omega$ and choose $h=h^{2} \in V \backslash\{\emptyset\}$ with $\alpha_{h}=\alpha$. Suppose $\alpha \in \operatorname{dom}(f)$. Then $(\alpha)(f b)=\left(\alpha_{f}\right) b=\alpha_{f \phi}$. Since $h f \neq \emptyset$, we have $\alpha_{h \phi} \in \operatorname{dom}(f \phi)$ and therefore $(\alpha)[b(f \phi)]=\left(\alpha_{h \phi}\right)(f \phi)=\alpha_{f \phi}$. Thus $(\alpha)(f b)=(\alpha)[b(f \phi)]$. If $\alpha \notin \operatorname{dom}(f)$, then $h f=\emptyset$ and so $\alpha_{h \phi} \notin \operatorname{dom}(f \phi)$ and so $\alpha \notin \operatorname{dom}(b(f \phi))$. It follows that $b(f \phi)=f b$ and so $f \phi=b^{-1} f b$. Therefore $\phi \in \operatorname{Inn}(V)$, as required.

Lemma 2.2. Let $U$ be a subsemigroup of $P_{\Omega}$ such that for all $\alpha \in \Omega$ there exists a constant idempotent in $U$ with value $\alpha$. Then $\operatorname{Aut}(U)=\operatorname{Inn}(U)$.

PROOF. Let $V$ denote the set of all constants in $U$ and let $V_{\beta}=\{f \in V \mid$ $\operatorname{im}(f) \subseteq\{\beta\}\}$ for every $\beta \in \Omega$. Thus $V=\cup_{\beta \in \Omega} V_{\beta}$. Take any $\beta \in \Omega$. By assumption, $V_{\beta} \backslash\{\emptyset\}$ contains an idempotent, and it is easy to check that $V_{\beta}$ is a 0 -minimal left ideal of $U$. It follows that $V$ is a subsemigroup of $U$. If $I$ is a 0 minimal left ideal of $U$ containing an idempotent $f \in V \backslash\{\emptyset\}$ with value $\beta \in \Omega$, then $\{\emptyset\} \neq V_{\beta}=V_{\beta} f \subseteq I$ which implies $I=V_{\beta}$. Clearly, if $\psi$ is an automorphism of a semigroup $S$ with zero and $J$ is a 0-minimal left ideal of $S$ containing a nonzero idempotent $e$, then $J \psi$ is also a 0-minimal left ideal of $S$ containing a nonzero idempotent $e \psi$. It follows that $V \phi=V$ for all $\phi \in \operatorname{Aut}(U)$.

Let $\phi \in \operatorname{Aut}(U)$. Then $\left.\phi\right|_{V} \in \operatorname{Aut}(V)$ and so, by Lemma 2.1, $\left.\phi\right|_{V} \in \operatorname{Inn}(V)$. Thus there exists $b \in S_{\Omega}$ such that $\left(\alpha_{f}\right) b=\alpha_{\left.f \phi\right|_{V}}$ and $\left.f \phi\right|_{V}=b^{-1} f b$ for all $f \in V \backslash\{\emptyset\}$. Take an arbitrary $g \in U$. Clearly, $\emptyset \phi=b^{-1} \emptyset b$. Suppose $g \neq \emptyset$. For each $\beta \in \Omega$, fix an idempotent $f_{\beta} \in V_{\beta} \backslash\{\emptyset\}$, so that $\alpha_{f_{\beta}}=\beta$. Then for $\beta \in \Omega$ we 
have,

$$
\begin{aligned}
\beta \in \operatorname{dom}(g) & \Longleftrightarrow\left(f_{\beta} \phi\right)(g \phi) \neq \emptyset \quad \Longleftrightarrow \alpha_{f_{\beta} \phi} \in \operatorname{dom}(g \phi) \\
& \Longleftrightarrow\left(\alpha_{f_{\beta}}\right) b \in \operatorname{dom}(g \phi) \Longleftrightarrow \beta \in(\operatorname{dom}(g \phi)) b^{-1},
\end{aligned}
$$

from which it follows that $\operatorname{dom}(g \phi)=(\operatorname{dom}(g)) b$ and hence

$$
\beta \in \operatorname{dom}(b(g \phi)) \Longleftrightarrow \beta b \in \operatorname{dom}(g) b \Longleftrightarrow \beta \in \operatorname{dom}(g)=\operatorname{dom}(g b) .
$$

Therefore $\operatorname{dom}(b(g \phi))=\operatorname{dom}(g b)$ and for each $\beta \in \operatorname{dom}(b(g \phi))$,

$$
\begin{array}{r}
(\beta)(b(g \phi))=\left(\left(\alpha_{f_{\beta}}\right) b\right) g \phi=\left(\alpha_{f_{\beta} \phi}\right) g \phi=\alpha_{\left(f_{\beta} \phi\right)(g \phi)}=\alpha_{\left(f_{\beta} g\right) \phi}=\left(\alpha_{f_{\beta} g}\right) b \\
=\left(\left(\alpha_{f_{\beta}}\right) g\right) b=(\beta)(g b),
\end{array}
$$

so $b(g \phi)=g b$ and so $g \phi=b^{-1} g b$. Thus $\phi=\phi_{b}$ and since $(U) \phi_{b}=U \phi=U$, we have $\phi \in \operatorname{Inn}(U)$.

Proof of TheOrem 1.1. The assumptions about the semigroup $U$ from the statement of the theorem will be used below without comment. By Lemma 2.2, $\operatorname{Aut}(U)=\operatorname{Inn}(U)$. Our goal is to prove that $\operatorname{Inn}(U)=\operatorname{Inn}_{\mathrm{Aut}_{H}\left(\rho^{\prime}\right)}(U)$. Since it is obvious that $\operatorname{Inn}_{\operatorname{Aut}_{H}\left(\rho^{\prime}\right)}(U) \leq \operatorname{Inn}(U)$, we only need to show that $\operatorname{Inn}(U) \leq$ $\operatorname{Inn}_{\mathrm{Aut}_{H}\left(\rho^{\prime}\right)}(U)$. Take any $\phi_{b} \in \operatorname{Inn}(U)$. Thus $b \in S_{\Omega}$ and $(U) \phi_{b}=(U) \phi_{b^{-1}}=U$. We are going to prove that $b \in \operatorname{Aut}_{H}\left(\rho^{\prime}\right)$.

Suppose $b \notin \operatorname{Aut}_{H}\left(\rho^{\prime}\right)$, that is, there exists $\left(\alpha_{1}, \ldots, \alpha_{m}\right) \in \rho^{\prime}$ such that $\left(\alpha_{1 t}, \ldots, \alpha_{m t}\right)^{b} \notin \rho^{\prime}$ for all $t \in H$. Let $\left(\beta_{1}, \ldots, \beta_{m}\right) \in \rho^{\prime}$. Then there exists $f \in U$ such that $\left(\beta_{1}, \ldots, \beta_{m}\right)^{f}=\left(\alpha_{1}, \ldots, \alpha_{m}\right)$. If $\left(\beta_{1 t_{0}}, \ldots, \beta_{m t_{0}}\right)^{b} \in \rho^{\prime}$ for some $t_{0} \in H$, then

$$
\left[\left(\beta_{1 t_{0}}, \ldots, \beta_{m t_{0}}\right)^{b}\right]^{b^{-1} f b}=\left(\beta_{1 t_{0}}, \ldots, \beta_{m t_{0}}\right)^{f b}=\left(\alpha_{1 t_{0}}, \ldots, \alpha_{m t_{0}}\right)^{b} \notin \rho^{\prime},
$$

so that $(f) \phi_{b} \notin U$, a contradiction. It follows that $\left(\beta_{1 t}, \ldots, \beta_{m t}\right)^{b} \notin \rho^{\prime}$ for all $t \in H$, and therefore $\left(\beta_{1}, \ldots, \beta_{m}\right)^{b} \in N(\rho, H)$. If $\left(\beta_{1}, \ldots, \beta_{m}\right)^{b^{-1}} \in \rho^{\prime}$, then there exists $g \in U$ such that $\left[\left(\beta_{1}, \ldots, \beta_{m}\right)^{b^{-1}}\right]^{g}=\left(\beta_{1}, \ldots, \beta_{m}\right)$, which implies $\left(\beta_{1}, \ldots, \beta_{m}\right)^{b^{-1} g b}=\left(\beta_{1}, \ldots, \beta_{m}\right)^{b} \notin \rho^{\prime}$ and so $(g) \phi_{b} \notin U ;$ a contradiction. We conclude that $\left(\beta_{1}, \ldots, \beta_{m}\right)^{b^{-1}} \notin \rho^{\prime}$. Since $\left(\beta_{1}, \ldots, \beta_{m}\right)^{b} \in N(\rho, H)$, there exists $h \in$ $U$ such that $\left[\left(\beta_{1}, \ldots, \beta_{m}\right)^{b}\right]^{h}=\left(\beta_{1}, \ldots, \beta_{m}\right)$, and therefore $\left(\beta_{1}, \ldots, \beta_{m}\right)^{b h b^{-1}}=$ $\left(\beta_{1}, \ldots, \beta_{m}\right)^{b^{-1}} \notin \rho^{\prime}$ and so $(h) \phi_{b^{-1}} \notin U$, again a contradiction.

Finally, let $a, b \in \operatorname{Aut}_{H}\left(\rho^{\prime}\right)$ such that $a \neq b$ and $\phi_{a}, \phi_{b} \in \operatorname{Aut}(U)$. It remains to prove that $\phi_{a} \neq \phi_{b}$. But there exists $\alpha \in \Omega$ such that $(\alpha) a \neq(\alpha) b$ and so $\phi_{a}$ and $\phi_{b}$ differ on any constant idempotent in $U$ with value $\alpha$. 


\section{Corollaries and Examples}

Note that if $\rho$ in Theorem 1.1 satisfies $\rho^{\prime}=\emptyset$ and $U$ is a subsemigroup of PEnd $(\rho)$ satisfying (1) in Theorem 1.1, then Theorem 1.1 offers no new information regarding $\operatorname{Aut}(U)$. That is, as there are no tuples of distinct elements in $\rho, U$ vacuously acts transitively from $N(\rho, H) \cup \rho^{\prime}$ to $\rho^{\prime}$. Moreover, in this case $\operatorname{Aut}_{H}\left(\rho^{\prime}\right)=S_{\Omega}$ and so Theorem 1.1 reasserts that $\operatorname{Aut}(U)=\operatorname{Inn}(U)$. However, this conclusion can be derived from the much weaker Lemma 2.2.

The following is a useful corollary of Theorem 1.1, which we will use later in the paper.

Corollary 3.1. Let $\rho$ be an $m$-ary relation on a finite set $\Omega$ for some $m \in \mathbb{N}$, let $H$ be a subgroup of $S_{m}$, and let $U \in\{\operatorname{PEnd}(\rho), \operatorname{End}(\rho), \operatorname{IEnd}(\rho)\}$ such that the following hold:

(1) $U$ contains a constant idempotent with value $\alpha$ for all $\alpha \in \Omega$;

(2) $U$ acts transitively from $N(\rho, H) \cup \rho^{\prime}$ to $\rho^{\prime}$;

(3) $\operatorname{Aut}_{H}\left(\rho^{\prime}\right)=\operatorname{Aut}_{H}(\rho)$.

Then $\operatorname{Aut}(U)=\left\{\phi_{a} \mid a \in \operatorname{Aut}_{H}(\rho)\right\} \cong \operatorname{Aut}_{H}(\rho)$.

ProOF. We prove the corollary in the case that $U=\operatorname{End}(\rho)$, the remaining cases can be proved analogously.

It follows from Theorem 1.1 and (3) that $\operatorname{Aut}(U)=\operatorname{Inn}_{\operatorname{Aut}_{H}(\rho)}(U)$. Let $a \in \operatorname{Aut}_{H}(\rho)$. We will prove that $a^{-1} U a=U$. Let $f \in \operatorname{End}(\rho)$ be arbitrary and let $\left(\alpha_{1}, \ldots, \alpha_{m}\right) \in \rho$. Since $a \in \operatorname{Aut}_{H}(\rho)$, there exists $t \in H$ such that $\left(\alpha_{1 t^{-1}}, \ldots, \alpha_{m t^{-1}}\right)^{a^{-1}} \in \rho$. Hence, since $f \in U,\left(\alpha_{1 t^{-1}}, \ldots, \alpha_{m t^{-1}}\right)^{a^{-1} f} \in \rho$ and so $\left(\alpha_{1}, \ldots, \alpha_{m}\right)^{a^{-1} f a} \in \rho$. Therefore $a^{-1} f a \in U$ and so $a^{-1} U a=U$, as required.

It follows that $\operatorname{Aut}(U)=\left\{\phi_{a} \mid a \in \operatorname{Aut}_{H}(\rho)\right\}$. Let $F: \operatorname{Aut}_{H}(\rho) \longrightarrow \operatorname{Aut}(U)$ be defined by $(a) F=\phi_{a}$. Then, since distinct elements in $\operatorname{Aut}_{H}(\rho)$ induce distinct automorphisms of $U$ and $\operatorname{Aut}(U)$ is finite, $F$ is a bijection. It is straightforward to verify that $F$ is a homomorphism, and the corollary follows.

Corollary 3.2. Let $\rho$ be a reflexive binary relation on a finite set $\Omega$ and let $H$ be a subgroup of $S_{2}$. Then $\operatorname{Aut}_{H}\left(\rho^{\prime}\right)=\operatorname{Aut}_{H}(\rho)$.

Proof. Since $\rho^{\prime} \subseteq \rho$, it follows that $\operatorname{Aut}_{H}(\rho) \leq \operatorname{Aut}_{H}\left(\rho^{\prime}\right)$. To prove the converse, let $a \in \operatorname{Aut}_{H}\left(\rho^{\prime}\right)$ and let $(\alpha, \beta) \in \rho$ be arbitrary. If $\alpha \neq \beta$, then either $(\alpha, \beta)^{a} \in \rho^{\prime} \subseteq \rho$ or $(\beta, \alpha)^{a} \in \rho^{\prime} \subseteq \rho$, as required. If $\alpha=\beta$, then, since $\rho$ is reflexive, $(\alpha, \beta)^{a}=(\beta, \alpha)^{a} \in \rho$. Hence $a \in \operatorname{Aut}_{H}(\rho)$ and $\operatorname{sod}_{\operatorname{Aut}_{H}}\left(\rho^{\prime}\right)=\operatorname{Aut}_{H}(\rho)$. 
Let $U, \rho$, and $H$ be as in Theorem 1.1. Then the following example demonstrates that there can exist elements $a \in \operatorname{Aut}_{H}\left(\rho^{\prime}\right)$ such that $a^{-1} U a \neq U$.

Example 3.3. Let $\Omega=\{1,2,3,4,5\}$, let $H=S_{5}$, and let

$$
\begin{array}{r}
\rho=\{(1,2,3,4,5),(2,1,3,4,5)\} \cup\left\{\left(\alpha_{1}, \alpha_{2}, \ldots, \alpha_{5}\right) \mid\left\{\alpha_{1}, \alpha_{2}, \ldots, \alpha_{5}\right\}=\{3,4\}\right\} \\
\cup\{(\alpha, \alpha, \alpha, \alpha, \alpha) \mid \alpha \in\{1, \ldots, 5\}\} .
\end{array}
$$

Then $N(\rho, H)=\emptyset, \operatorname{Aut}\left(\rho^{\prime}\right)=S_{\{1,2\}}=\operatorname{Aut}(\rho), S_{\{1,2\}} \times S_{\{3,4,5\}}$ is a subgroup of $\operatorname{Aut}_{H}\left(\rho^{\prime}\right)$, and every element of $\operatorname{Aut}_{H}(\rho)$ stabilizes $\{3,4\}$ setwise. In particular, $\operatorname{Aut}_{H}\left(\rho^{\prime}\right) \neq \operatorname{Aut}_{H}(\rho)$.

Let $U=\operatorname{Aut}(\rho) \cup\left\{f \in T_{5} \mid \operatorname{im}(f)=\{3,4\}\right.$ or $\left.|\operatorname{im}(f)|=1\right\}$. Then $U$ is a subsemigroup of $\operatorname{PEnd}(\rho)$ and, since $\operatorname{Aut}(\rho)$ is transitive from $\rho^{\prime}$ to $\rho^{\prime}, U$ is also transitive from $\rho^{\prime}$ to $\rho^{\prime}$. Let

$$
f=\left(\begin{array}{lllll}
1 & 2 & 3 & 4 & 5 \\
3 & 3 & 3 & 3 & 4
\end{array}\right) \in U .
$$

Then $(3,4,3,4,3)^{(45) f(45)}=(3,5,3,5,3) \notin \rho$. Hence $(45) f(45) \notin U$ and so the element $(45) \in \operatorname{Aut}_{H}\left(\rho^{\prime}\right)$ does not induce an inner automorphism of $U$.

The following example shows that it is not true that if $a \in \operatorname{Aut}_{H}\left(\rho^{\prime}\right)$ and $a^{-1} U a=U$, then $a \in \operatorname{Aut}_{H}(\rho)$.

Example 3.4. Let $\rho$ be the relation from Example 3.3 and let $V=\operatorname{Aut}(\rho) \cup$ $\left\{f \in T_{5} \mid f\right.$ is constant $\}$. Then, as in Example 3.3, $V$ is transitive on $\rho^{\prime}$. However, $(45) \in \operatorname{Aut}_{H}\left(\rho^{\prime}\right)$ and $(45) V(45)=V$ but $(45) \notin \operatorname{Aut}_{H}(\rho)$, as required.

\section{Applications I - Transformation semigroups}

In this section we apply Theorem 1.1 to determine the automorphism groups of several well-known transformation semigroups, defined below. Some of the results contained in this section are well-known and included here only to illustrate how Theorem 1.1 can be used. Recall that $T_{\Omega}, P_{\Omega}$, and $I_{\Omega}$ denote the monoids of all total mappings, all partial mappings, and all partial injective mappings of the finite set $\Omega$, respectively. As above, if $\Omega=\{1,2, \ldots, m\}$, then we may write $T_{m}, P_{m}$, or $I_{m}$ instead of $T_{\Omega}, P_{\Omega}$, or $I_{\Omega}$, respectively.

Corollary 4.1. Let $\Omega$ be a finite set and let $U$ be any one of the semigroups $P_{\Omega}, T_{\Omega}$, and $I_{\Omega}$. Then $\operatorname{Aut}(U)=\operatorname{Inn}(U)=\left\{\phi_{a} \mid a \in S_{\Omega}\right\} \cong S_{\Omega}$. 
PROOF. Let $\rho=\Omega \times \Omega$. Then

$$
\operatorname{PEnd}(\rho)=P_{\Omega}, \operatorname{End}(\rho)=T_{\Omega}, \text { and } \operatorname{IEnd}(\rho)=I_{\Omega} .
$$

In any case, $U$ contains a constant idempotent with value $\alpha$ for all $\alpha \in \Omega$ and so part (1) of the hypothesis of Corollary 3.1 is satisfied. If $H$ is an arbitrary subgroup of $S_{2}$, then $N(\rho, H)=\emptyset$. Also $\rho^{\prime}=(\Omega \times \Omega) \backslash\{(\alpha, \alpha) \mid \alpha \in \Omega\}$. It is clear that $U$ is transitive from $\rho^{\prime}$ to $\rho^{\prime}$ and so part (2) of Corollary 3.1 is satisfied. Finally, $\rho$ is a reflexive binary relation and so by Corollary 3.2, $\operatorname{Aut}_{H}\left(\rho^{\prime}\right)=\operatorname{Aut}_{H}(\rho)$. Thus, by Corollary 3.1, $\operatorname{Aut}(U)=\left\{\phi_{a} \mid a \in S_{\Omega}\right\} \cong S_{\Omega}$, as required.

Next, we consider a set $\Omega$ with $m n$ elements and an equivalence relation $\rho$ on $\Omega$ with $m$ classes each of size $n$. We aim to describe the groups of automorphisms of the monoids $\operatorname{PEnd}(\rho), \operatorname{End}(\rho)$ and $\operatorname{IEnd}(\rho)$.

First, recall that if $S$ and $T$ are semigroups acting on sets $\Gamma$ and $\Sigma$, respectively, then the wreath product of $S$ and $T$, denoted $S \imath T$, is the set $S \times T^{\Gamma}$, where $T^{\Gamma}$ denotes the set of all mappings from $\Gamma$ to $T$, with multiplication

$$
(s, f)(t, g)=\left(s t, f \star^{s} g\right),
$$

where $(\alpha)^{s} g=(\alpha s) g$ and $(\alpha) f \star^{s} g=(\alpha) f \cdot(\alpha)^{s} g$, for all $\alpha \in \Gamma$. The semigroup $S \imath T$ acts on $\Gamma \times \Sigma$ as follows: $(\alpha, \sigma)^{(s, f)}=(\alpha s,(\sigma)(\alpha f))$, for all $(\alpha, \sigma) \in \Gamma \times \Sigma$ and $(s, f) \in S \times T^{\Gamma}$. For further details about wreath products see [15] or [17].

Now, observe that $\operatorname{Aut}(\rho) \cong S_{m} \prec S_{n}$ and $\operatorname{End}(\rho) \cong T_{m} \imath T_{n}$ (for a proof, see [4, Lemma 2.1]). Furthermore, we have:

Corollary 4.2. Let $\rho$ be an equivalence relation on a set $\Omega$ with $m$ classes each of size $n$ and $\operatorname{let} U \in\{\operatorname{PEnd}(\rho), \operatorname{End}(\rho), \operatorname{IEnd}(\rho)\}$. Then $\operatorname{Aut}(U)=\left\{\phi_{a} \mid a \in\right.$ $S_{m}\left\langle S_{n}\right\} \cong S_{m}\left\langle S_{n}\right.$.

PROOF. First, notice that $U$ contains a constant idempotent with value $\alpha$ for all $\alpha \in \Omega$.

Let $H$ be the trivial subgroup of $S_{2}$. Then, since $\rho$ is symmetric, $N(\rho, H)$ contains all the pairs of distinct elements in $\Omega^{2} \backslash \rho$. Next, we prove that $U$ is transitive from the pairs of distinct entries in $\Omega^{2}$ to $\rho^{\prime}$. Let $(\alpha, \beta) \in \Omega^{2}$ and let $(\gamma, \delta) \in \rho$ such that $\alpha \neq \beta$ and $\gamma \neq \delta$. Then

$$
f=\left(\begin{array}{ll}
\alpha & \beta \\
\gamma & \delta
\end{array}\right)
$$


is an element of $\operatorname{IEnd}(\rho)$, and so of $\operatorname{PEnd}(\rho)$, such that $(\alpha, \beta)^{f}=(\gamma, \delta)$. Let $g: \Omega \longrightarrow \Omega$ be any total mapping with image $\{\gamma, \delta\}$ such that $\alpha g=\gamma$ and $\beta g=\delta$. Then $g \in \operatorname{End}(\rho)$ and again $(\alpha, \beta)^{g}=(\gamma, \delta)$.

Finally, by Corollaries 3.1 and 3.2, we deduce that that $\operatorname{Aut}(U)=\left\{\phi_{a} \mid a \in\right.$ $S_{m}\left\{S_{n}\right\} \cong S_{m}\left\{S_{n}\right.$.

In particular, by the above observation, we have that $\operatorname{Aut}\left(T_{m} \prec T_{n}\right) \cong S_{m} \prec S_{n}$.

\section{Applications II - Ordered sets}

In this section, we consider the automorphism groups of semigroups of order-preserving partial mappings of a partially ordered set, and some related semigroups.

Theorem 5.1. Let $\Omega$ be a finite set, let $\rho$ be a partial order on $\Omega$, and let $U \in$ $\{\operatorname{PEnd}(\rho), \operatorname{End}(\rho), \operatorname{IEnd}(\rho)\}$. Then $\operatorname{Aut}(U)=\left\{\phi_{a} \mid a \in \operatorname{Aut}_{S_{2}}(\rho)\right\} \cong \operatorname{Aut}_{S_{2}}(\rho)$ where the latter is the group of automorphisms and anti-automorphisms of $\rho$.

PROOF. If $\alpha, \beta \in \Omega$ are such that $(\alpha, \beta) \notin \rho$ and $(\beta, \alpha) \notin \rho$, then we will write $\alpha \| \beta$. An anti-chain in $\rho$ is any subset $\Sigma$ of $\Omega$ where $\alpha \| \beta$ for all $\alpha, \beta \in \Sigma$ with $\alpha \neq \beta$.

Since $\rho$ is reflexive, $\operatorname{PEnd}(\rho), \operatorname{End}(\rho)$, and $\operatorname{IEnd}(\rho)$ contain a constant idempotent with value $\alpha$ for all $\alpha \in \Omega$. If $H=S_{2}$, then

$$
N(\rho, H)=\left\{(\alpha, \beta) \in \Omega^{2} \mid \alpha \| \beta\right\} .
$$

We will prove that $U$ is transitive from $N(\rho, H) \cup \rho^{\prime}$ to $\rho^{\prime}$.

If $(\beta, \alpha) \notin \rho, \beta \neq \alpha$, and $(\gamma, \delta) \in \rho$, then the mapping $f$ from the proof of Corollary 4.2 is an element of $\operatorname{IEnd}(\rho)$ and $\operatorname{PEnd}(\rho)$ such that $(\alpha, \beta)^{f}=(\gamma, \delta)$.

It remains to prove that $U$ is transitive from $N(\rho, H) \cup \rho^{\prime}$ to $\rho^{\prime}$ when $U=$ $\operatorname{End}(\rho)$. Let $\Sigma$ be a subset of $\Omega$. Then we define

$$
\Sigma^{\wedge}=\left\{\beta \in \Omega \mid(\forall \alpha \in \Sigma)(\alpha, \beta) \in \rho^{\prime} \text { or } \beta \| \alpha\right\}
$$

and

$$
\Sigma^{\vee}=\left\{\beta \in \Omega \mid(\forall \alpha \in \Sigma)(\beta, \alpha) \in \rho^{\prime} \text { or } \beta \| \alpha\right\}
$$

If $\Sigma$ is a maximal (with respect to containment) anti-chain in $\rho$, then the sets $\Sigma^{\wedge}$, $\Sigma$, and $\Sigma^{\vee}$ are disjoint and their union is the whole of $\Omega$. 
Let $\alpha, \beta, \gamma, \delta \in \Omega$ be such that $\alpha \neq \beta,(\beta, \alpha) \notin \rho$, and $(\gamma, \delta) \in \rho^{\prime}$. If $(\alpha, \beta) \in \rho$, then since $\Omega$ is finite, there exists a maximal anti-chain $\Sigma$ in $\rho$ such that $\alpha \in \Sigma$. It follows that $\beta \in \Sigma^{\wedge}$. Let $f: \Omega \longrightarrow \Omega$ be defined by

$$
\epsilon f= \begin{cases}\gamma & \epsilon \in \Sigma \cup \Sigma^{\vee} \\ \delta & \epsilon \in \Sigma^{\wedge} .\end{cases}
$$

Then $f \in \operatorname{End}(\rho)$ and $(\alpha, \beta)^{f}=(\gamma, \delta)$.

If $(\alpha, \beta) \notin \rho$, then as in the previous case, since $\Omega$ is finite, there exists a maximal anti-chain $\Sigma$ in $\rho$ such that $\alpha, \beta \in \Sigma$. Let $f: \Omega \longrightarrow \Omega$ be defined by

$$
\epsilon f= \begin{cases}\gamma & \epsilon \in(\Sigma \backslash\{\beta\}) \cup \Sigma^{\vee} \\ \delta & \epsilon \in \Sigma^{\wedge} \cup\{\beta\} .\end{cases}
$$

Then $f \in \operatorname{End}(\rho)$ and $(\alpha, \beta)^{f}=(\gamma, \delta)$.

In any case, $U$ is transitive from $N(\rho, H) \cup \rho^{\prime}$ to $\rho^{\prime}$. Thus, by Corollaries 3.1 and 3.2, $\operatorname{Aut}(U)=\left\{\phi_{a} \mid a \in \operatorname{Aut}_{S_{2}}(\rho)\right\} \cong \operatorname{Aut}_{S_{2}}(\rho)$, as required.

If $\rho$ is the usual total order of $\{1, \ldots, n\}$, then $\operatorname{End}(\rho), \operatorname{PEnd}(\rho)$, and $\operatorname{IEnd}(\rho)$ are usually denoted $\mathcal{O}_{n}, \mathcal{P O} \mathcal{O}_{n}, \mathcal{P O \mathcal { I } _ { n }}$ (the semigroups of total, partial, and partial injective order-preserving mappings of the chain $\rho$, respectively). These monoids have been extensively studied, for example see [1, 2, 8, 13, 14].

Recall that, for a given real number $x$, the expressions $\lfloor x\rfloor$ and $\lceil x\rceil$ denote the greatest integer less than or equal to $x$ and the least integer greater than or equal to $x$, respectively. We also let $C_{2}$ denote a cyclic group of order 2 .

The following is an immediate corollary of Theorem 5.1.

Corollary 5.2. If $U \in\left\{\mathcal{O}_{n}, \mathcal{P} \mathcal{O}_{n}, \mathcal{P O} \mathcal{I}_{n}\right\}$, then

$$
\operatorname{Aut}(U)=\left\langle\phi_{(1 n)(2 n-1) \cdots(\lfloor n / 2\rfloor\lceil n / 2\rceil+1)}\right\rangle \cong C_{2} .
$$

Let $\mathcal{O} \mathcal{D}_{n}$, let $\mathcal{P O D}{ }_{n}$, and let $\mathcal{P O D \mathcal { I } _ { n }}$ be the monoids of all total, partial, and partial injective order-preserving and order-reversing mappings of the usual total order on $\{1, \ldots, n\}$, respectively. Again these monoids appear in several papers in the literature, for example see $[9,10,11]$. The automorphism groups of these monoids are given in the theorem below. However, the theorem is not a direct corollary of any of the preceding theorems, since these semigroups are not defined as the partial endomorphisms of a relation.

We require the following well-known combinatorial fact in order to find the automorphism groups of $\mathcal{O D}_{n}, \mathcal{P O D}{ }_{n}$, and $\mathcal{P O D \mathcal { I } _ { n }}$. 
Lemma 5.3. If $p, q \in \mathbb{N}$, then in any sequence of distinct natural numbers of length $p q+1$, there exists a strictly increasing subsequence of length $p$ or a strictly decreasing subsequence of length $q$.

Theorem 5.4. Let $n \geq 10$ and let $U \in\left\{\mathcal{O D}_{n}, \mathcal{P O D}{ }_{n}, \mathcal{P O D} \mathcal{I}_{n}\right\}$. Then

$$
\operatorname{Aut}(U)=\left\langle\phi_{(1 n)(2 n-1) \cdots(\lfloor n / 2\rfloor\lceil n / 2\rceil+1)}\right\rangle \cong C_{2} .
$$

PROOF. We prove the theorem in the case of $U=\mathcal{O D}_{n}$; the the cases when $U=\mathcal{P O} \mathcal{D}_{n}$, or $\mathcal{P O D} \mathcal{I}_{n}$ follow by an analogous argument.

Let $\rho$ be the ternary relation on $\{1, \ldots, n\}$ defined by

$$
\rho=\left\{\left(\alpha_{1}, \alpha_{2}, \alpha_{3}\right) \mid \alpha_{1} \leq \alpha_{2} \leq \alpha_{3} \text { or } \alpha_{1} \geq \alpha_{2} \geq \alpha_{3}\right\} .
$$

Then $U=\mathcal{O D}_{n} \leq \operatorname{End}(\rho)$. It is straightforward to verify that $U$ is transitive from $\rho^{\prime} \cup N\left(\rho, S_{3}\right)=\rho^{\prime}$ to $\rho^{\prime}$, and $U$ contains a constant idempotent with value $\alpha$ for all $\alpha \in\{1,2, \ldots, n\}$. It follows from Theorem 1.1 that $\operatorname{Aut}(U)=\operatorname{Inn}_{\text {Aut }_{S_{3}}\left(\rho^{\prime}\right)}(U)$.

We will show that $\operatorname{Aut}_{S_{3}}\left(\rho^{\prime}\right)=\operatorname{Aut}_{S_{3}}(\rho)=\operatorname{Aut}(\rho)$ and

$$
\operatorname{Aut}(\rho)=\langle(1 n)(2 n-1) \cdots(\lfloor n / 2\rfloor\lceil n / 2\rceil+1)\rangle .
$$

Since $\operatorname{Aut}_{S_{3}}(\rho)$ is always a subset of $\operatorname{Aut}_{S_{3}}\left(\rho^{\prime}\right)$, to prove that $\operatorname{Aut}_{S_{3}}\left(\rho^{\prime}\right)=$ $\operatorname{Aut}_{S_{3}}(\rho)$ it suffices to show that $\operatorname{Aut}_{S_{3}}\left(\rho^{\prime}\right) \leq \operatorname{Aut}_{S_{3}}(\rho)$. Let $a \in \operatorname{Aut}_{S_{3}}\left(\rho^{\prime}\right)$ and let $\left(\alpha_{1}, \alpha_{2}, \alpha_{3}\right) \in \rho$. Then there exists $\left(\beta_{1}, \beta_{2}, \beta_{3}\right) \in \rho^{\prime}$ such that $\left\{\alpha_{1}, \alpha_{2}, \alpha_{3}\right\} \subseteq$ $\left\{\beta_{1}, \beta_{2}, \beta_{3}\right\}$. Since $a \in \operatorname{Aut}_{S_{3}}\left(\rho^{\prime}\right)$, there exists $t \in S_{3}$ such that $\left(\beta_{1 t}, \beta_{2 t}, \beta_{3 t}\right)^{a} \in \rho$. Hence $\left(\alpha_{1 t}, \alpha_{2 t}, \alpha_{3 t}\right)^{a} \in \rho$ and so $a \in \operatorname{Aut}_{S_{3}}(\rho)$. We have shown that $\operatorname{Aut}_{S_{3}}\left(\rho^{\prime}\right) \leq$ $\operatorname{Aut}_{S_{3}}(\rho)$.

Clearly, $\operatorname{Aut}(\rho) \leq \operatorname{Aut}_{S_{3}}(\rho)$. To prove the converse inclusion, take an arbitrary $a \in \operatorname{Aut}_{S_{3}}(\rho)$. Then there exists $t \in S_{3}$ such that $\left(\alpha_{1 t}, \alpha_{2 t}, \alpha_{3 t}\right)^{a} \in \rho$ for all $\left(\alpha_{1}, \alpha_{2}, \alpha_{3}\right) \in \rho$. By Lemma 5.3, since $10=3 \cdot 3+1$, the sequence $1 a, 2 a, \ldots, 10 a$ must contain a subsequence $\beta_{1} a, \beta_{2} a, \beta_{3} a$ where $\beta_{1}<\beta_{2}<\beta_{3}$ and $\beta_{1} a<\beta_{2} a<\beta_{3} a$ or $\beta_{1} a>\beta_{2} a>\beta_{3} a$. Therefore $\left(\beta_{1}, \beta_{2}, \beta_{3}\right) \in \rho,\left(\beta_{1}, \beta_{2}, \beta_{3}\right)^{a} \in \rho$ and $\left(\beta_{1 t}, \beta_{2 t}, \beta_{3 t}\right)^{a} \in \rho$. This implies that $t$ is the identity permutation or $t=(13)$. In either case, by the definition of $\rho$, we have that $a \in \operatorname{Aut}(\rho)$. $\operatorname{Thus}^{\operatorname{Aut}_{S_{3}}}(\rho)=$ $\operatorname{Aut}(\rho)$.

Let $a \in \operatorname{Aut}(\rho)$. We will prove that $1 a \in\{1, n\}$. Assume to the contrary that $1 a \notin\{1, n\}$. If $n a \neq 1$, then $\left(1,1 a^{-1}, n\right) \in \rho$ but $\left(1,1 a^{-1}, n\right)^{a}=(1 a, 1, n a) \notin \rho$, a contradiction. Therefore $n a=1$. Now, we have that $\left(1, n a^{-1}, n\right) \in \rho$ and $\left(1, n a^{-1}, n\right)^{a}=(1 a, n, n a)=(1 a, n, 1) \notin \rho$ since $1 a \neq n$, a contradiction. Hence $1 a \in\{1, n\}$. 
If $1 a=1$ and $\alpha, \beta \in\{2, \ldots, n\}$ are such that $\alpha<\beta$, then $(1, \alpha, \beta) \in \rho$ and so $(1, \alpha a, \beta a) \in \rho$. Hence $\alpha a<\beta a$ and so $a$ is the identity on $\{1,2, \ldots, n\}$. If $1 a=n$ and $\alpha, \beta \in\{2, \ldots, n\}$ are such that $\alpha<\beta$, then $(1, \alpha, \beta) \in \rho$ and so $(n, \alpha a, \beta a) \in \rho$. Thus $\alpha a>\beta a$ and so $a=(1 n)(2 n-1) \cdots(\lfloor n / 2\rfloor\lceil n / 2\rceil+1)$. Therefore

$$
\operatorname{Aut}(\rho)=\langle(1 n)(2 n-1) \cdots(\lfloor n / 2\rfloor\lceil n / 2\rceil+1)\rangle .
$$

It is straightforward to verify that $\phi_{a}(U)=U$ for all $a \in \operatorname{Aut}(\rho)$ and so

$$
\begin{aligned}
\operatorname{Aut}(U) & =\left\{\phi_{a} \mid a \in \operatorname{Aut}_{S_{3}}\left(\rho^{\prime}\right) \text { and } \phi_{a}(U)=U\right\}=\left\{\phi_{a} \mid a \in \operatorname{Aut}(\rho)\right\} \\
& =\left\langle\phi_{(1 n)(2 n-1) \cdots(\lfloor n / 2\rfloor\lceil n / 2\rceil+1)}\right\rangle \cong C_{2},
\end{aligned}
$$

as required.

A finite sequence $\left(\alpha_{1}, \alpha_{2}, \ldots, \alpha_{m}\right)$ of natural numbers is called cyclic if there exists $k \geq 1$ such that $\alpha_{1 g^{k}} \leq \alpha_{2 g^{k}} \leq \cdots \leq \alpha_{m g^{k}}$, where $g=(12 \cdots m)$. A partial mapping with domain and range contained in $\{1,2, \ldots, n\}$ is orientationpreserving if the image of every cyclic sequence in $\operatorname{dom}(f)$ is a cyclic sequence. A finite sequence $\left(\alpha_{1}, \alpha_{2}, \ldots, \alpha_{m}\right)$ of natural numbers is called anti-cyclic if there exists $k \geq 1$ such that $\alpha_{1 g^{k}} \geq \alpha_{2 g^{k}} \geq \cdots \geq \alpha_{m g^{k}}$, where $g=(12 \cdots m)$. If $f$ is an orientation-preserving mapping, then it is straightforward to verify that the image of every anti-cyclic sequence in $\operatorname{dom}(f)$ is anti-cyclic.

Let $\mathcal{O P}{ }_{n}$, let $\mathcal{P O P}{ }_{n}$, and let $\mathcal{P O P \mathcal { I } _ { n }}$ be the monoids of all total, partial, and partial injective orientation-preserving mappings, respectively, of the set $\{1,2, \ldots, n\}$. Some references from the literature concerning these monoids are $[6,7,9,10,12,16]$.

For $n \in \mathbb{N}$, denote the dihedral group of order $2 n$ by $D_{2 n}$. It is well-known that $D_{2 n}$ is isomorphic to the subgroup of the symmetric group $S_{n}$ generated by the permutations: $(12 \cdots n)$ and $(1 n)(2 n-1) \cdots(\lfloor n / 2\rfloor\lceil n / 2\rceil+1)$.

Theorem 5.5. Let $U \in\left\{\mathcal{O P}_{n}, \mathcal{P O P} \mathcal{P}_{n}, \mathcal{P O P} \mathcal{I}_{n}\right\}$. Then

$$
\operatorname{Aut}(U)=\left\langle\phi_{(12 \cdots n)}, \phi_{(1 n)(2 n-1) \cdots(\lfloor n / 2\rfloor\lceil n / 2\rceil+1)}\right\rangle \cong D_{2 n} .
$$

PROOF. We prove the theorem in the case that $U=\mathcal{O P}_{n}$; the other two cases follow by analogous arguments. Since $\mathcal{O}_{n} \leq \mathcal{O P}{ }_{n}$, it follows that $\mathcal{O P}{ }_{n}$ contains a constant idempotent with value $\alpha$ for all $\alpha \in\{1,2, \ldots, n\}$.

Let $\rho$ denote the set of all cyclic sequences of length 3 over $\{1,2, \ldots, n\}$ and let $H=\langle(13)\rangle \leq S_{3}$. Then $\mathcal{O P}_{n} \leq \operatorname{End}(\rho)$ and $N(\rho, H)=\emptyset$. We will prove that 
$\mathcal{O P}{ }_{n}$ acts transitively from $\rho^{\prime}$ to $\rho^{\prime}$. Let $(i, j, k),\left(i^{\prime}, j^{\prime}, k^{\prime}\right) \in \rho^{\prime}$ be arbitrary. Then either $i<j<k, j<k<i$ or $k<i<j$. If $i<j<k$, then define

$$
f=\left(\begin{array}{ccccccccccc}
1 & \cdots & i-1 & i & i+1 & \cdots & j-1 & j & j+1 & \cdots & n \\
i^{\prime} & \cdots & i^{\prime} & i^{\prime} & j^{\prime} & \cdots & j^{\prime} & j^{\prime} & k^{\prime} & \cdots & k^{\prime}
\end{array}\right) .
$$

If $j<k<i$, then define

$$
f=\left(\begin{array}{ccccccccccc}
1 & \cdots & j-1 & j & j+1 & \cdots & k-1 & k & k+1 & \cdots & n \\
j^{\prime} & \cdots & j^{\prime} & j^{\prime} & k^{\prime} & \cdots & k^{\prime} & k^{\prime} & i^{\prime} & \cdots & i^{\prime}
\end{array}\right) .
$$

If $k<i<j$, then define

$$
f=\left(\begin{array}{ccccccccccc}
1 & \cdots & k-1 & k & k+1 & \cdots & i-1 & i & i+1 & \cdots & n \\
k^{\prime} & \cdots & k^{\prime} & k^{\prime} & i^{\prime} & \cdots & i^{\prime} & i^{\prime} & j^{\prime} & \cdots & j^{\prime}
\end{array}\right) .
$$

In any case, $f \in \mathcal{O} \mathcal{P}_{n}$ and $(i, j, k)^{f}=\left(i^{\prime}, j^{\prime}, k^{\prime}\right)$. It follows from Theorem 1.1 that $\operatorname{Aut}\left(\mathcal{O P}_{n}\right)=\operatorname{Inn}_{\operatorname{Aut}_{H}\left(\rho^{\prime}\right)}\left(\mathcal{O P}_{n}\right)$.

We will prove that $\operatorname{Aut}_{H}\left(\rho^{\prime}\right)=\operatorname{Aut}_{H}(\rho)$ and

$$
\operatorname{Aut}_{H}(\rho)=\langle(12 \cdots n),(1 n)(2 n-1) \cdots(\lfloor n / 2\rfloor\lceil n / 2\rceil+1)\rangle .
$$

As before, we have that $\operatorname{Aut}_{H}(\rho) \leq \operatorname{Aut}_{H}\left(\rho^{\prime}\right)$. Let $a \in \operatorname{Aut}_{H}\left(\rho^{\prime}\right)$ and let $\left(\alpha_{1}, \alpha_{2}, \alpha_{3}\right) \in \rho$ be arbitrary. If $\left(\alpha_{1}, \alpha_{2}, \alpha_{3}\right) \in \rho \backslash \rho^{\prime}$, then $\left(\alpha_{1 t}, \alpha_{2 t}, \alpha_{3 t}\right)^{a}$ lies in $\rho \backslash \rho^{\prime}$ for all $t \in S_{3}$, since every triple with at most two distinct elements is cyclic and hence in $\rho$. If $\left(\alpha_{1}, \alpha_{2}, \alpha_{3}\right) \in \rho^{\prime}$ then, since $a \in \operatorname{Aut}_{H}\left(\rho^{\prime}\right)$, there exists $t \in H$ such that $\left(\alpha_{1 t}, \alpha_{2 t}, \alpha_{3 t}\right)^{a} \in \rho$. Hence $a \in \operatorname{Aut}_{H}(\rho)$ and $\operatorname{so}_{\operatorname{Aut}_{H}}\left(\rho^{\prime}\right)=\operatorname{Aut}_{H}(\rho)$.

Let $a \in \operatorname{Aut}_{H}(\rho)$. Then there exists $t \in H=\{1,(13)\}$ such that $\left(\alpha_{1}, \alpha_{2}, \alpha_{3}\right) \in$ $\rho$ implies $\left(\alpha_{1 t}, \alpha_{2 t}, \alpha_{3 t}\right)^{a} \in \rho$. By multiplying $a$ by an appropriate power of $(12 \cdots n)$ we may assume that $1 a=1$.

If $t$ is the identity permutation and $\alpha, \beta \in\{1,2, \ldots, n\}$ such that $1<\alpha<$ $\beta \leq n$, then $(1, \alpha, \beta) \in \rho$ and so $(1, \alpha a, \beta a) \in \rho$. Hence $\alpha a<\beta a$ and so $a$ is the identity of $\operatorname{Aut}_{H}(\rho)$.

If $t=(13)$ and $\alpha, \beta \in\{1,2, \ldots, n\}$ such that $1<\alpha<\beta \leq n$, then $(1, \alpha, \beta) \in$ $\rho$ and so $(\beta a, \alpha a, 1) \in \rho$. It follows that $\beta a<\alpha a$ and so

$$
a=(1 n)(2 n-1) \cdots(\lfloor n / 2\rfloor\lceil n / 2\rceil+1) .
$$

It is easy to verify that $\phi_{a}\left(\mathcal{O P}_{n}\right)=\mathcal{O P}{ }_{n}$ for all $a \in \operatorname{Aut}(\rho)$ and so

$$
\begin{aligned}
\operatorname{Aut}\left(\mathcal{O P}_{n}\right) & =\left\{\phi_{a} \mid a \in \operatorname{Aut}_{H}\left(\rho^{\prime}\right) \text { and } \phi_{a}\left(\mathcal{O P}_{n}\right)=\mathcal{O P}_{n}\right\}=\left\{\phi_{a} \mid a \in \operatorname{Aut}_{H}(\rho)\right\} \\
& =\left\langle\phi_{(12 \cdots n)}, \phi_{(1 n)(2 n-1) \cdots(\lfloor n / 2\rfloor\lceil n / 2\rceil+1)} \cong \cong D_{2 n} .\right.
\end{aligned}
$$


A partial mapping with domain and range contained in $\{1,2, \ldots, n\}$ is called orientation-reversing if the image of every cyclic sequence in $\operatorname{dom}(f)$ is an anticyclic sequence.

Let $\mathcal{O} \mathcal{R}_{n}$, let $\mathcal{P O} \mathcal{R}_{n}$, and let $\mathcal{P O} \mathcal{O} \mathcal{I}_{n}$ be the monoids of all total, partial, and partial injective orientation-preserving and orientation-reversing mappings, respectively, of the set $\{1,2, \ldots, n\}$. For further details of the known results concerning these monoids see $[5,9,10,12]$.

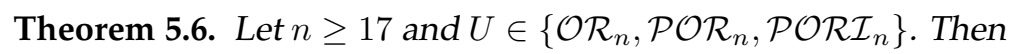

$$
\operatorname{Aut}(U)=\left\langle\phi_{(12 \cdots n)}, \phi_{(1 n)(2 n-1) \cdots(\lfloor n / 2\rfloor\lceil n / 2\rceil+1)}\right\rangle \cong D_{2 n} .
$$

PROOF. As in the proofs of the previous theorems, we only prove the case when $U=\mathcal{O R}_{n}$, since the proofs of the others cases are analogously.

Let $\rho$ be the set of quadruples of elements from $\{1,2, \ldots, n\}$ that are cyclic, anti-cyclic, or contain at most two distinct elements. It is straightforward to see that $\mathcal{O R}_{n} \leq \operatorname{End}(\rho), \mathcal{O} \mathcal{R}_{n}$ contains a contains a constant idempotent with value $\alpha$ for all $\alpha \in\{1,2, \ldots, n\}$, and $N\left(\rho, S_{4}\right)=\emptyset$. It follows using a similar argument as in the proof of Theorem 5.5 that $\mathcal{O} \mathcal{R}_{n}$ acts transitively from $\rho^{\prime}$ to $\rho^{\prime}$. It follows by Theorem 1.1 that $\operatorname{Aut}\left(\mathcal{O} \mathcal{R}_{n}\right)=\operatorname{Inn}_{\mathrm{Aut}_{S_{4}}\left(\rho^{\prime}\right)}\left(\mathcal{O} \mathcal{R}_{n}\right)$.

We will show that $\operatorname{Aut}_{S_{4}}\left(\rho^{\prime}\right)=\operatorname{Aut}(\rho)$ and

$$
\operatorname{Aut}(\rho)=\langle(12 \cdots n),(1 n)(2 n-1) \cdots(\lfloor n / 2\rfloor\lceil n / 2\rceil+1)\rangle .
$$

Since $\operatorname{Aut}(\rho) \leq \operatorname{Aut}_{S_{4}}\left(\rho^{\prime}\right)$ always holds, it suffices to show that $\operatorname{Aut}_{S_{4}}\left(\rho^{\prime}\right) \leq$ $\operatorname{Aut}\left(\rho^{\prime}\right)$ and $\operatorname{Aut}\left(\rho^{\prime}\right) \leq \operatorname{Aut}(\rho)$.

Let $a \in \operatorname{Aut}_{S_{4}}\left(\rho^{\prime}\right)$. Then there exists $t \in S_{4}$ such that $\left(\alpha_{1 t}, \alpha_{2 t}, \alpha_{3 t}, \alpha_{4 t}\right)^{a} \in$ $\rho^{\prime}$ for all $\left(\alpha_{1}, \alpha_{2}, \alpha_{3}, \alpha_{4}\right) \in \rho^{\prime}$. By Lemma 5.3, since $17=4 \cdot 4+1$, the sequence $(1 a, \ldots, 17 a)$ must contain a subsequence $\left(\beta_{1} a, \beta_{2} a, \beta_{3} a, \beta_{4} a\right)$ where either $\beta_{1} a<\beta_{2} a<\beta_{3} a<\beta_{4} a$ or $\beta_{1} a>\beta_{2} a>\beta_{3} a>\beta_{4} a$. In either case, $\left(\beta_{1}, \beta_{2}, \beta_{3}, \beta_{4}\right),\left(\beta_{1}, \beta_{2}, \beta_{3}, \beta_{4}\right)^{a},\left(\beta_{1 t}, \beta_{2 t}, \beta_{3 t}, \beta_{4 t}\right)^{a} \in \rho^{\prime}$. In other words, $t$ is the identity or $t=(14)(23)$. Therefore

$$
\left(\alpha_{1 t^{-1}}, \alpha_{2 t^{-1}}, \alpha_{3 t^{-1}}, \alpha_{4 t^{-1}}\right) \in \rho^{\prime}
$$

for all $\left(\alpha_{1}, \alpha_{2}, \alpha_{3}, \alpha_{4}\right) \in \rho^{\prime}$ and so

$$
\left(\alpha_{1}, \alpha_{2}, \alpha_{3}, \alpha_{4}\right)^{a}=\left(\alpha_{1 t^{-1} t}, \alpha_{2 t^{-1} t}, \alpha_{3 t^{-1} t}, \alpha_{4 t^{-1} t}\right)^{a} \in \rho^{\prime} .
$$

Thus $a \in \operatorname{Aut}\left(\rho^{\prime}\right)$ and so $\operatorname{Aut}_{S_{4}}\left(\rho^{\prime}\right) \leq \operatorname{Aut}\left(\rho^{\prime}\right)$. 
We will now prove that $\operatorname{Aut}\left(\rho^{\prime}\right) \leq \operatorname{Aut}(\rho)$. Let $a \in \operatorname{Aut}\left(\rho^{\prime}\right)$ and let $\alpha$ be any quadruple in $\rho \backslash \rho^{\prime}$. If $\alpha$ contains at most 2 distinct elements, then $\alpha^{a}$ contains at most 2 distinct elements and so $\alpha^{a} \in \rho$. If $\alpha$ contains 3 distinct elements, then there exists $\beta \in \rho^{\prime}$ containing all the elements occurring in $\alpha$. Hence $\beta^{a} \in \rho^{\prime}$ and so $\beta^{a}$ is cyclic or anti-cyclic. It follows that $\alpha^{a}$ is cyclic or anti-cyclic, or in other words, that $\alpha^{a} \in \rho$. Hence $a \in \operatorname{Aut}(\rho)$ and so $\operatorname{Aut}\left(\rho^{\prime}\right) \leq \operatorname{Aut}(\rho)$.

It is easy to verify that

$$
\langle(12 \cdots n),(1 n)(2 n-1) \cdots(\lfloor n / 2\rfloor\lceil n / 2\rceil+1)\rangle \leq \operatorname{Aut}(\rho) .
$$

Let $a \in \operatorname{Aut}(\rho)$. Then by multiplying by a power of $(12 \cdots n)$ we may assume that $1 a=1$. Seeking a contradiction assume that $2 a \notin\{2, n\}$. Since $\left(1,2, n a^{-1}, n\right) \in \rho$, if $n a \neq n$, then $\left(1,2, n a^{-1}, n\right)^{a}=(1,2 a, n, n a) \notin \rho$. It follows that $n a=n$ and so $\left(1,2,2 a^{-1}, n\right)^{a}=(1,2 a, 2, n) \notin \rho$, but $\left(1,2,2 a^{-1}, n\right) \in \rho$ and so we have a contradiction.

It follows that $2 a \in\{2, n\}$. Using a similar argument, we can deduce that $n a \in\{2, n\}$. By multiplying by $(2 n)(3 n-1) \cdots(\lfloor(n+1) / 2\rfloor\lceil(n+1) / 2\rceil+1) \in$ $\operatorname{Aut}(\rho)$, if necessary, we may suppose that $2 a=n$ and $n a=2$. If $2<\alpha<\beta<n$, then $(2, \alpha, \beta, n) \in \rho$ and so $(2, \alpha, \beta, n)^{a}=(n, \alpha a, \beta a, 2) \in \rho$. It follows that $\alpha a>$ $\beta a$ which implies that

$$
\begin{aligned}
a=(2 n)(3 n-1) \cdots( & (\lfloor(n+1) / 2\rfloor\lceil(n+1) / 2\rceil+1) \\
& \quad\langle\langle(12 \cdots n),(1 n)(2 n-1) \cdots(\lfloor n / 2\rfloor\lceil n / 2\rceil+1)\rangle,
\end{aligned}
$$

as required.

It is easy to verify that $\phi_{a}\left(\mathcal{O} \mathcal{R}_{n}\right)=\mathcal{O} \mathcal{R}_{n}$ for all $a \in \operatorname{Aut}(\rho)$ and so

$$
\begin{aligned}
\operatorname{Aut}\left(\mathcal{O} \mathcal{R}_{n}\right) & =\left\{\phi_{a} \mid a \in \operatorname{Aut}_{S_{4}}\left(\rho^{\prime}\right) \text { and } \phi_{a}\left(\mathcal{O} \mathcal{R}_{n}\right)=\mathcal{O} \mathcal{R}_{n}\right\}=\left\{\phi_{a} \mid a \in \operatorname{Aut}(\rho)\right\} \\
& =\left\langle\phi_{(12 \cdots n)}, \phi_{(1 n)(2 n-1) \cdots(\lfloor n / 2\rfloor\lceil n / 2\rceil+1)}\right\rangle \cong D_{2 n} .
\end{aligned}
$$

Acknowledgement. The research presented in this paper was partially supported by the FCT and FEDER project ISFL-1-143 of Centro de Álgebra da Universidade de Lisboa, and by the FCT and PIDDAC project PTDC/MAT/69514/2006.

\section{References}

[1] A. Ya. Aǔzenštat, The defining relations of the endomorphism semigroup of a finite linearly ordered set, Sibirsk. Mat. 3 (1962), 161-169 (Russian). 
[2] A. Ya. Aǐzenštat, Homomorphisms of semigroups of endomorphisms of ordered sets, Uch. Zap., Leningr. Gos. Pedagog. Inst. 238 (1962), 38-48 (Russian).

[3] J. Araújo and J. Konieczny, A method of finding automorphism groups of endomorphism monoids of relational systems, Discrete Math. 307 (2007), 1609-1620.

[4] J. Araújo and C. Schneider, The rank of the endomorphism monoid of a uniform partition, Semigroup Forum 78 (2009), 498-510.

[5] R. E. Arthur and N. Ruškuc, Presentations for two extensions of the monoid of order-preserving mappings on a finite chain, Southeast Asian Bull. Math. 24 (2000), 1-7.

[6] P. M. Catarino and P. M. Higgins, The monoid of orientation-preserving mappings on a chain, Semigroup Forum 58 (1999), 190-206.

[7] V. H. Fernandes, The monoid of all injective orientation preserving partial transformations on a finite chain, Comm. Algebra 28 (2000), 3401-3426.

[8] V. H. Fernandes, The monoid of all injective order preserving partial transformations on a finite chain, Semigroup Forum 62 (2001), 178-204.

[9] V. H. Fernandes, G. M. S. Gomes and M. M. Jesus, Presentations for some monoids of injective partial transformations on a finite chain, Southeast Asian Bull. Math. 28 (2004), 903-918.

[10] V. H. Fernandes, G. M. S. Gomes and M. M. Jesus, Presentations for some monoids of partial transformations on a finite chain, Comm. Algebra 33 (2005), 587-604.

[11] V. H. Fernandes, G. M. S. Gomes and M. M. Jesus, Congruences on monoids of order-preserving or order-reversing transformations on a finite chain, Glasgow Mathematical Journal 47 (2005), 413424 .

[12] V. H. Fernandes, G. M. S. Gomes and M. M. Jesus, Congruences on monoids of transformations preserving the orientation on a finite chain, J. Algebra 321 (2009), 743-757.

[13] G. M. S. Gomes and J. M. Howie, On the ranks of certain semigroups of order-preserving transformations, Semigroup Forum 45 (1992), 272-282.

[14] J. M. Howie, Product of idempotents in certain semigroups of transformations, Proc. Edinburgh Math. Soc. 17 (1971), 223-236.

[15] G. Lallement, Semigroups and combinatorial applications, Pure and Applied Mathematics, A Wiley-Interscience Publication, John Wiley \& Sons, New York-Chichester-Brisbane, 1979.

[16] D. B. McAlister, Semigroups generated by a group and an idempotent, Comm. Algebra 26 (1998), 515-547.

[17] J. D. P. Meldrum, Wreath products of groups and semigroups, Pitman Monographs and Surveys in Pure and Applied Mathematics, Longman, Harlow, 1995.

DEPARTAMENTO DE MATEMÁTICA DA UNIVERSIDADE ABERTA, RUA DA ESCOLA POLITÉCNICA, 147, 1269-001 LISBOA, PORTUGAL; AND CENTRO DE ÁlGEBRA DA UNIVERSIDADE DE LISBOA, AV. PROF. GAMA PINTO, 2, 1649-003 LISBOA, PORTUGAL

E-mail: mjoao@ptmat.fc.ul.pt

DEPARTAMENTO DE MATEMÁTICA DA FACULDADE DE CIÊNCIAS E TECNOLOGIA DA UNIVERSIDADE NOVA DE LISBOA, QUINTA DA TORRE, 2829-516 CAPARICA, PORTUGAL; AND CENTRO DE Álgebra dA UNIVERSIDAde DE LISBOA, AV. PROF. GAMA PINTO, 2, 1649-003 LisbOA, PORTUGAL

E-mail: vhf@fct.unl.pt

DEPARTAMENTO DE MATEMÁTICA DA FACULDADE DE CIÊNCIAS E TECNOLOGIA DA UNIVERSIDADE NOVA DE LISBOA, QUINTA DA TORRE, 2829-516 CAPARICA, PORTUGAL; AND CENTRO DE Álgebra DA UNIVERSidAde DE LISBOA, AV. PROF. GAMA PINTO, 2, 1649-003 LISBOA, PORTUGAL 
E-mail: messias@ptmat.fc.ul.pt

MATHEMATICAL INSTITUTE, ST ANDREWS UNIVERSiTY, NORTH HAUGH, ST ANDREWS, FIFE, UK, KY16 9SS

E-mail: victor@mcs.st-and.ac.uk

MATHEMATICAL INSTITUTE, ST ANDREWS UNIVERSITY, NORTH HAUGH, ST ANDREWS, FIFE, UK, KY16 9SS

E-mail: jdm3@st-and.ac.uk 